\title{
EDITORIAL
}

\section{Coronary flow: clinical considerations}

\section{V Cokkinos, A Manginas, V Voudris}

In the measurement of coronary blood flow to determine the success of percutaneous coronary intervention, invasive techniques, coupled with plaque characterisation and other intracoronary imaging modalities, may prove invaluable

T he measurement of coronary blood flow (CBF) has transcended the realm of observational curiosity through the emergence of two developments:

- Firstly, the application of easier methods to measure it, which have replaced the older cumbersome inert gas and thermodilution techniques. Two notable examples are the noninvasive positron emission tomography (PET) calculations and the intracoronary Doppler measurements of flow velocity. Formerly, Doppler catheters were used, which could not measure flow distally to a stenosis. This problem was overcome by the use of the Flowire, which with a diameter of 0.014 inches can be placed across the stenosis during the course of invasive procedures. Because of its small diameter, this wire does not cause significant flow disturbances, as was the case with the previously used Doppler catheters. The Doppler wire actually measures flow velocity. For the velocity values to be equivalent to $\mathrm{CBF}$, the cross sectional area of the vessel must be measured.

- Secondly, the realisation that diminution of flow distally to a significant stenosis is reliably correlated to manifestations of ischaemia.

Concomitantly with the measurement of $\mathrm{CBF}$, the importance of the coronary flow reserve (CFR) was appreciated. This term signifies the difference between CBF under maximal hyperaemia, produced by various interventions, and basal flow. Basal CBF amounts to approximately $1 \mathrm{ml} / \mathrm{g}$ of myocardial tissue/min; at maximal hyperaemia it can increase by 3-4 times, according to the technique employed.

Many factors influence the CFR values. Because of the use of CFR in the assessment of coronary artery stenosis, the degree of stenosis has attained major importance. However, the actual coronary resistance is determined to a far greater degree by microvascular resistance. Since the assessment of microvascular resistance, as will be discussed later, is difficult, the concept of the relative coronary flow reserve (RCFR) has emerged, in which the CFR of the involved artery is compared to an adjacent normal artery. Thus each patient is his own control. A not sufficiently appreciated element is that a high basal CBF amounts to a lower CFR. This old concept is "dis- covered" anew whenever a new technique measuring coronary flow emerges.

Gould and his team showed in 1974 in the dog that while basal coronary flow remains normal until a stenosis $\geqslant 90 \%$ is produced, hyperaemia is abolished above a coronary artery diameter stenosis $\geqslant 60-70 \%$. ${ }^{1}$ This finding, later documented with PET measurements, clarified the mechanisms of effort angina; during physical exercise the pressure-rate product, which corresponds closely to myocardial oxygen consumption, can increase up to three times, while CBF does not increase.

When CBF is assessed to characterise the impact of a coronary stenotic lesion, a single measurement does not suffice. According to Kern, a significant lesion is associated with one or more of the following abnormalities: a post-stenotic absolute CFR $<2.0$, RCFR $<0.9$, proximal to distal flow velocity ratio $<1.7$, diastolic to systolic velocity ratio $<1.8$, and when the fractional flow reserve (FFR) is used, a value $<0.75$. $^{2}$

FFR is another index used to assess the functional significance of coronary stenoses. It is calculated as the ratio of the pressure distal to the stenosis during maximal hyperaemia to the aortic pressure, measured by a pressure sensor mounted on a 0.014 inch guide wire. Values $<0.75$ are considered haemodynamically significant.

Correlations of CFR with ischaemic manifestations were prompt to emerge. Wilson and colleagues $^{3}$ showed that at exercise electrocardiography, the degree of ST depression corresponded significantly with the CFR but not the severity of epicardial artery stenosis. Bartunek and colleagues ${ }^{4}$ using dobutamine echocardiography also showed that wall motion abnormalities emerged with a diameter stenosis $\sim 60 \%$ and an FFR $\sim 0.67$. Our group was the first to show that the degree of $\mathrm{Tl}-201$ reversibility correlated very strongly with the difference of CFR between a normal artery and an adjacent artery with a significant stenosis. ${ }^{5}$

We were also able to offer some insight into why adenosine (and its equivalent dipyridamole) are preferred over dobutamine for the pharmacologic stress of Tl-201 scintigraphy. We showed

Abbreviations: CFR, coronary flow reserve; $C B F$, coronary blood flow; DEBATE, Doppler endpoints balloon angioplasty trial Europe; DESTINI, Doppler endpoint stenting international investigation; FFR, fractional flow reserve; LAD, left anterior descending; LIMA, left internal mammary artery; $\mathrm{PCl}$, percutaneous coronary intervention PET, positron emission tomography; RCFR, relative coronary flow reserve; SPECT, single photon emission computed tomography; TIMI, thrombolysis in myocardial infarction 
that adenosine produces flow inhomogeneity to a greater degree than maximal dose dobutamine alone. ${ }^{6}$

FFR values $<0.75$ have also been correlated with ischaemia, both at exercise testing and myocardial perfusion imaging. $^{7}$

A question which arises when CBF is measured is whether the large epicardial arteries or the microvasculature are at fault. The main disease entities associated with microvascular disease are a previous myocardial infarction, left ventricular hypertrophy, diabetes mellitus, the syndrome $\mathrm{X}$ and others. The pressure wire cannot address this question. A low CFR in the presence of normal FFR is a practical index of microvascular dysfunction. Very recently Pijls and colleagues ${ }^{8}$ combined $^{2}$ FFR and CFR by coronary thermodilution to facilitate assessment of microvascular disease.

\section{PREDICTIVE VALUE OF CFR ON EARLY AND LATE FUTURE EVENTS AND ANGIOGRAPHIC RESTENOSIS}

The DEBATE trial evaluated the effect of balloon angioplasty on distal CFR in relation to the clinical outcome during a six month follow up period. ${ }^{9}$ Postprocedure CFR was found to have a modest prognostic value in predicting the incidence of symptoms and/or ischaemia at four weeks. When anatomic and physiologic data were combined, a distal CFR after balloon angioplasty $>2.5$ with a residual diameter stenosis $\leqslant 35 \%$ identified lesions with a low incidence of recurrence of symptoms at one month and six months, a low need for reintervention, and a lower restenosis rate compared with patients who did not meet these criteria. In the DEBATE II study, a low CFR postprocedure was an independent predictor of major adverse cardiac events at 30 days and at one year. ${ }^{10}$

The DESTINI study ${ }^{11}$ indicated that, compared with elective stenting $(\mathrm{n}=370$ patients), provisional stenting $(\mathrm{n}=218$ lesions) based on CFR and angiographic criteria can avoid the costs and complications of stenting in about $50 \%$ of patients with no compromise in clinical or angiographic outcomes.

Bech and colleague ${ }^{12}$ analysed the prognostic significance of FFR in patients undergoing balloon angioplasty. The two year event-free survival for patients with postprocedural diameter stenosis $\leqslant 35 \%$ and FFR $\geqslant 0.90$ was significantly better than that for patients with suboptimal values of either of these variables. FFR immediately after stenting was the most significant independent variable related to major adverse cardiac events, including the need for repeat target vessel revascularisation at six months.

An inverse situation was also considered by $\operatorname{Kern}^{13}$ in the evaluation of CBF measurements and whether it could be used to defer a percutaneous coronary intervention (PCI). He found a low rate of clinical events $(<10 \%)$ over a two year follow up period in intermediate stenoses with a normal CFR. Very recently Chamuleau and colleagues ${ }^{14}$ found that a CFR $<2.0$ was a significant predictor of events-stronger than Tl-201 SPECT. Actually they found few events $(1 / 21)$ at one year follow up in patients with a positive SPECT and a normal CFR. We do not know how many clinicians would decide to measure CFR rather than proceed to PCI in a patient with a strongly positive SPECT who is found to have a $60 \%$ lesion at angiography.

\section{MYOCARDIAL VIABILITY HIBERNATION}

For the last nine years, studies following acute myocardial reperfusion either by thrombolysis or percutaneous interventions after an acute occlusion are associated with appreciable coronary flow abnormalities. CFR gives a measure of the anticipated myocardial recovery. Gibson and colleagues ${ }^{15}$ showed that improved epicardial flow at 90 minutes after thrombolysis was associated with improved survival. Stone and associates ${ }^{16}$ reported the same results with primary or rescue angioplasty.
Gibson and colleagues ${ }^{17}$ were the first to advance another entity in CBF, the tissue level perfusion assessed by the myocardial perfusion grade, which is expressed by the "myocardial blush".

Myocardial hibernation refers to a state of chronic ischaemic ventricular dysfunction characterised by improvement after coronary revascularisation. By definition, viability of the cardiomyocytes is preserved as a result of metabolic downregulation, an important adaptive mechanism against myocardial necrosis. A controversy exists as regards the reduction of resting myocardial flow in hibernating segments and its clinical impact, reflected also by the use of various terms such as "chronic stunning", and "short term" and "long term hibernation". In a meta-analysis published in 1999, Schwartz and colleagues ${ }^{18}$ did not find convincing evidence supporting the widely accepted notion of chronic basal flow reduction in hibernating myocardium.

It appears that the differences in various studies are due to observations of a progressive pathophysiological phenomenon at various points along its natural course. During the initial stages the resting blood flow is not necessarily reduced, the post-revascularisation contractile recovery is complete, but the CFR in the hibernating segments is clearly diminished (early hibernation). ${ }^{19}{ }^{20}$ As coronary stenoses progress, the episodes of ischaemia and stunning become more frequent (repetitive stunning), gradually resulting in functional deterioration and possibly eventual depression of the resting myocardial flow, followed by cellular alterations and incomplete functional post-revascularisation recovery (late hibernation). ${ }^{21}$

\section{SIMPLER, INEXPENSIVE, NON-INVASIVE DIAGNOSTIC MODALITIES}

Flowire and the pressure wire techniques are costly and invasive. PET is non-invasive, but costly and not widely available. The Tl-201 washout rates have not been widely correlated nor widely used to assess CBF, and contrast echocardiography is only now coming of age for this purpose.

Transoesophageal echocardiography has been used for more than 10 years to measure CFR with consistently good correlations. However, the technique is cumbersome. Newer echocardiographic techniques have enabled the measurement of CBF by transthoracic echocardiography. Correlations with invasive techniques have proved very good.22 Results may further improve with contrast echocardiography.

A corresponding technique has been the Doppler measurement of flow in the left internal mammary artery (LIMA) grafted to the left anterior descending (LAD) coronary artery, from the left supraclavicular fossa. We have found that the CFR values with this technique correspond very closely $(r=0.88)$ with those obtained by use of the Flowire, and that a normal LIMA/LAD CFR has an excellent prognostic value. ${ }^{23}$

An invasive but inexpensive method was devised by Gibson and colleagues, ${ }^{24}$ the TIMI frame count-that is, the time required for contrast material to reach certain landmarks of coronary anatomy.

Our group was the first to show that CFR measured with the TIMI frame count after intravenous adenosine administration has an excellent correlation with that measured with the Flowire. ${ }^{25}$ We have also used the baseline TIMI frame count post-angioplasty administration to predict restenosis after PTCA. A ratio of a TIMI frame count/minimal lumen diameter was strongly predictive of restenosis. ${ }^{26}$ This was very recently verified by Gibson and colleagues ${ }^{27}$ for PTCA or directional atherectomy in acute coronary syndromes. If this index is found to be true for stenting also, this technique can provide a simple means to guide interventional efforts at the catheterisation table.

\section{THOUGHTS FOR THE FUTURE}

Conjecture is a fruitless pastime. However, we can venture some predictions. Non-invasive techniques will probably 
predominate in the coming years. Flow of the epicardial arteries by Doppler, microvascular function by contrast injection, and tissue viability by Doppler tissue imaging will be combined in one echocardiographic measurement. The same can probably be achieved by magnetic resonance imaging, which is costly and cumbersome however.

These two techniques will be increasingly and interchangeably used to assess success of PCI in acute-especially myocardial infarction-and chronic situations to examine tissue viability. Non-invasive CFR measurement reflecting microvascular function will direct and monitor medical treatment in unfavourable metabolic situations, such as scar tissue, the metabolic syndrome, diabetes mellitus, dyslipidaemia, hypertension, and dilated cardiomyopathy.

However, we believe that it is unwise to sing a premature requiem for the invasive techniques. If they are coupled with plaque thermography or other intracoronary imaging modalities such as optical coherence tomography or refined intravascular ultrasound systems, they may prove invaluable for early detection of the unstable plaque, an obsession of the current cardiologist comparable to the quest of the medieval knights for the holy grail.

\section{Authors' affiliations}

D V Cokkinos, University of Athens, Onassis Cardiac Surgery Center, Athens, Greece

A Manginas, V Voudris, Onassis Cardiac Surgery Center

\section{REFERENCES}

1 Gould KL, Lipscomb K, Hamilton GW. Physiologic basis for assessing critical coronary stenosis. Am J Cardiol 1974:33:87-94.

2 Kern MJ. Coronary physiology revisited: practical insights from the cardiac catheterization laboratory. Circulation 2000;101:1344-51.

3 Wilson RF. Assessment of the human coronary circulation using a Doppler catheter. Am J Cardiol 1991;67:44D-56D.

4 Bartunek J, Van Schuerbeeck E, de Bruyne B. Comparison of exercise electrocardiography and dobutamine echocardiography with invasively assessed myocardial fractional flow reserve in evaluation of severity of coronary arterial narrowing. Am J Cardiol 1997;79:478-81

5 Voudris V, Manginas A, Vassilikos V, et al. Coronary flow velocity changes after intravenous dypiridamole infusion: measurements using intravascular Doppler guide wire. A documentation of flow inhomogeneity. J Am Coll Cardiol 1996;27: 1 148-55.

6 Petropoulakis PN, Pavlides GS, Manginas AN, et al. Intracoronary flow velocity measurements in adjacent stenotic and normal coronary arteries during incremental intravenous dobutamine stress and intracoronary adenosine injection. Catheter Cardiovasc Interv 1999:48:1-9.

7 Pijls NHJ, Van Gelder B, Van der Voort P, et al. Fractional flow reserve: a useful index to evaluate the influence of an epicardial coronary stenosis on myocardial blood flow. Circulation 1995;92:3183-93.

8 Pijls NHJ, De Bruyne B, Smith L, et al. Coronary thermodilution to assess flow reserve validation in humans. Circulation 2002;105:2482-6.

9 Serruys PW, di Mario C, Piek JJ, et al. Prognostic value of intracoronary flow velocity and diameter stenosis in assessing the short- and long-term outcomes of coronary balloon angioplasty: the DEBATE study (Doppler endpoints balloon angioplasty trial Europe). Circulation 1997;96:3369-77

10 Serruys PW, de Bruyne B, Carlier S, et al. Randomized comparison of primary stenting and provisional balloon angioplasty guided by flow velocity measurement. Doppler endpoints balloon angioplasty trial Europe (DEBATE) II study group. Circulation 2000;102:2930-7.

11 Di Mario C, Moses JW, Anderson TJ, et al. Randomized comparison of elective stent implantation and coronary balloon angioplasty guided by online quantitative angiography and intracoronary Doppler. DESTIN study group (Doppler endpoint stenting international investigation). Circulation 2000; 102:2938-44

12 Bech GJW, Piils NHJ, de Bruyne B, et al. Usefulness of fractional flow reserve to predict clinical outcome after balloon angioplasty. Circulation 1999;99:883-8.

13 Kern MJ. Curriculum in interventional cardiology: coronary pressure and flow measuerments in the cardiac catheterization laboratory. Catheter Cardiovasc Interv 2001:54:378-400.

14 Chamuleau SAJ, Meuwissen M, van ECK-Smit BLF, et al. Fractional flow reserve, absolute and relative coronary blood flow velocity reserve in relation to the results of technetium- $99 \mathrm{~m}$ sestamibi single-photon emission somputed tomography in patients with two vessel coronary artery disease. J Am Coll Cardiol 2001;37:1316-22.

15 Gibson CM, Murphy SA, Rizzo M, et al. Relationship between TIMI frame count and clinical outcomes after thrombolytic administration. Circulation 1999;99:1945-50.

16 Stone GW, Peterson MA, Lansky AJ, et al. Impact of normalized myocardial perfusion after successful angioplasty in acute myocardial infarction. J Am Coll Cardiol 2002;39:591-7.

17 Gibson CM, Cannon CP, Murphy SA, et al. Relationship of TIMI myocardial perfusion grade to mortality after administration of thrombolytic drugs. Circulation 2000;101:125-30.

18 Schwarz ER, Speakman MT, vom Dahl J, et al. Hibernating myocardium is there evidence for chronic flow reduction? Heart Disease 1999;3: 155-62.

19 Heusch G, Schulz R. The biology of myocardial hibernation. Trends Cardiovasc Med 2000;10:108-14.

20 Camici PG, Wijns W, Borgers $M$, et al. Pathophysiological mechanisms of chronic reversible left ventricular dysfunction due to coronary artery disease (hibernating myocardium). Circulation 1997:96:3205-14.

21 Camici PG, Rimoldi O. Myocardial hibernation vs repetitive stunning in patients. Cardiol Rev 1999;7:39-43.

22 Cadollah S, Thaber KB, Kawanishi D, et al. Comparison of intracoronary Doppler guide wire and transesophageal ehcocardiography in measurement of flow velocity and coronary flow reserve in the left anterior descending coronary artery. Am Heart $J$ $1998 ; 135: 38-42$

23 Voudris V, Athanassopoulos G, Vassilikos V, et al. Usefulness of flow reserve in the left internal mammary artery to determine graft patency to the left anterior descending artery. Am J Cardiol 1999:83:1 157-63.

24 Gibson CM, Cannon CP, Daley WL, et al. The TIMl frame count method: a quantitative method of assessing coronary artery flow. Circulation 1996;93:879-88

25 Manginas A, Gatzov P, Chasikidis $\mathrm{CH}$, et al. Estimation of coronary flow reserve using the thrombolysis in myocardial infarction (TIMI) frame count method. Am J Cardiol 1999;83:1562-5.

26 Stankovic G, Manginas A, Voudris V, et al. Prediction of restenosis after coronary angioplasty by use of a new index: TIMI frame count/minimal luminal diameter ratio. Circulation 2000;101:962-8.

27 Gibson CM, Dotani MI, Murphy SA, et al, for the RESTORE Investigators. Correlates of coronary blood flow before and after percutaneous coronary intervention and their relationship to angiographic and clinical outcomes in the RESTORE trial. Am Heart J 2002;144:130-5. 